\title{
Cardiovascular prevention and rehabilitation for patients with ventricular assist device From exercise therapy to long-term therapy Part II: Long-term therapy
}

\author{
Prevenzione e riabilitazione cardiovascolare \\ in pazienti con device di assistenza ventricolare \\ Dall'esercizio alla terapia a lungo termine \\ Parte II: Terapia a lungo termine
}

\author{
Massimo Pistono1, Ugo Corrà1, Marco Gnemmi1, Alessandro Imparato1, Roberto Caruso1, \\ Gianluigi Balestroni², Franco Tarro Genta ${ }^{3}$, Elisabetta Angelino4, Pantaleo Giannuzzi ${ }^{1}$
}

\begin{abstract}
Cardiovascular prevention and rehabilitation for patients with ventricular assist device. From exercise therapy to long-term therapy. Part II: Long-term therapy. M. Pistono, U. Corrà, M. Gnemmi, A. Imparato, R. Caruso, G. Balestroni, F. Tarro Genta, E. Angelino, P. Giannuzzi.

Over the years left ventricular assist devices (VADs) have become more durable and reliable, smaller, simpler, easier to implant and more comfortable. The extensive experience now acquired shows successful hospital discharge with VAD use. We are entering an era in which long-term mechanical circulatory support will play an increasing role in the approach to end-stage heart failure (HF); at the same time, the extension of VADs into destination therapy has revealed the limitations of our understanding of these populations.
\end{abstract}

This second paper on cardiovascular prevention and rehabilitation for patients with left VADs will deal with the management of patients outside the highly specialized HF centers and surgical setting, with particular focus on postoperative patient management. Outpatient management of VAD patients is time-intensive, and a multidisciplinary approach is ideal in long-term care. Although the new devices have definite advantages over the older pumps, some challenges still remain, i.e. infection, stroke, device thrombosis, gastrointestinal bleeding, recurrent $\mathrm{HF}$ symptomatology with or without multisystem organ failure, and occurrence of ventricular arrhythmias.

Keywords: rehabilitation, ventricular assistance device.

Monaldi Arch Chest Dis 2011; 76: 136-145.

\footnotetext{
1 Division of Cardiology, Salvatore Maugeri Foundation, IRCCS, Veruno (NO), Italy.

2 Physchology Unit, Salvatore Maugeri Foundation, IRCCS, Veruno (NO), Italy.

3 Division of Cardiology, Presidio Major, Salvatore Maugeri Foundation, IRCCS, Torino, Italy.

4 Physchology Unit, Presidio Major, Salvatore Maugeri Foundation, IRCCS, Torino, Italy.
}

Corresponding author: Dr. Ugo Corrà; Divisione di Cardiologia, Laboratory for the Analysis of Cardio-Respiratory Signals, IRCCS Fondazione "S. Maugeri"; Via per Revislate, 13; I-28010 Veruno (NO), Italy; Phone 0039-0322-884711; Fax 0039-0322-830294; E-mail address: ugo.corrà@fsm

Over the years left ventricular assist devices (VADs) have become more durable and reliable, smaller, simpler, easier to implant and more comfortable [1-3]. Along with improvements in technology an extensive experience has now been gained with use of VADs showing that they lead to successful hospital discharge and outpatient management for periods of months to years, and significant recovery of functional status. The current approach in most centers is now to delay relisting of patients for heart transplantation (HT) until they have recovered physical condition and are stable [4]. We are entering an era in which long-term mechanical circulatory support is becoming a realistic alternative to HT and a life-line for the vast majority of advanced heart failure (HF) patients, ineligible for transplantation due to co-morbidities. However the extension of VADs from 'bridge' to 'destination' therapy has revealed limitations in our understanding of these populations.

This second manuscript on cardiovascular prevention and rehabilitation for patients with left VADs will deal with their management outside the highly specialized HF centers and surgical environment, with particular attention to long-term postoperative patient management. Outpatient management of VAD patients is time-intensive, and a multidisciplinary approach is recommended in long-term care. Although the new devices have several advantages over the older pumps, some challenges still remain, e.g. infection, stroke, device thrombosis, gastrointestinal bleeding, recurrent HF symptomatology with or without multisystem organ failure [3], and occurrence of ventricular arrhythmias. In addi- 
tion, a key point for success in terms of quality of life and survival is preparing the VAD patient for life outside of the hospital. Finally, we present here practical insights gained from our 3-year experience in management of VAD patients in our Rehabilitation Centers, while the candidacy criteria of VAD implantation, and early postoperative management are beyond the scope of this paper, and we refer the reader to dedicated reviews [5-6].

\section{Management of the outpatient on long-term left ventricular assist device support}

\section{Heart failure management after left ventricular assist device implant}

Heart failure management after left VAD implant should include the application of standard American College of Cardiology/American Heart Association (ACC/AHA) recommended evidencebased medications for HF - angiotension inhibitors and receptor blockers (ARB), beta-blockers, and aldosterone antagonists [7]. In addition to offering some patients the chance for myocardial recovery while on VAD support, these medications work with the VAD to reduce the activation of the renin-angiotensin-aldosterone system (RAAS) and sympathetic nervous system (SNS). The RAAS and SNS not only play a critical role in adverse myocardial remodeling that may impact long-term left and right ventricular performance, but they also drive fluid retention and increase systemic afterload. Hydralazine and nitrate combination therapy can be added to the medical regimen of patients who are on maximal tolerated doses of the above medications, particularly in the setting of elevated pulmonary vascular resistance or systemic hypertension.

Based on our experience, we have developed a graded pharmacological management plan to promote the prescription of evidence-based cardiovascular drugs in VAD patients during inpatient cardiac rehabilitation (figure 1); although VAD complications may mean that the plan has to be adapted, the general goal is to try and achieve the optimization of pharmacological therapy during hospital stay. Due to the fact that long-term medical and rehabilitation management of LVAD patient is in its infancy, we are conscious that this schematic and pragmatic "step up" clinical approach is open to criticisms.

\section{Hypertension}

With axial flow devices, conventional measurement of blood pressure (BP) is difficult, and it is common practice to measure mean BP by Doppler method. In the immediate postoperative period, an arterial line is used to follow BP. Once the patient is mobile and in the outpatient setting, Doppler measurement with a manual BP cuff is used by listening to the first Korotkoff sounds, assumed to represent

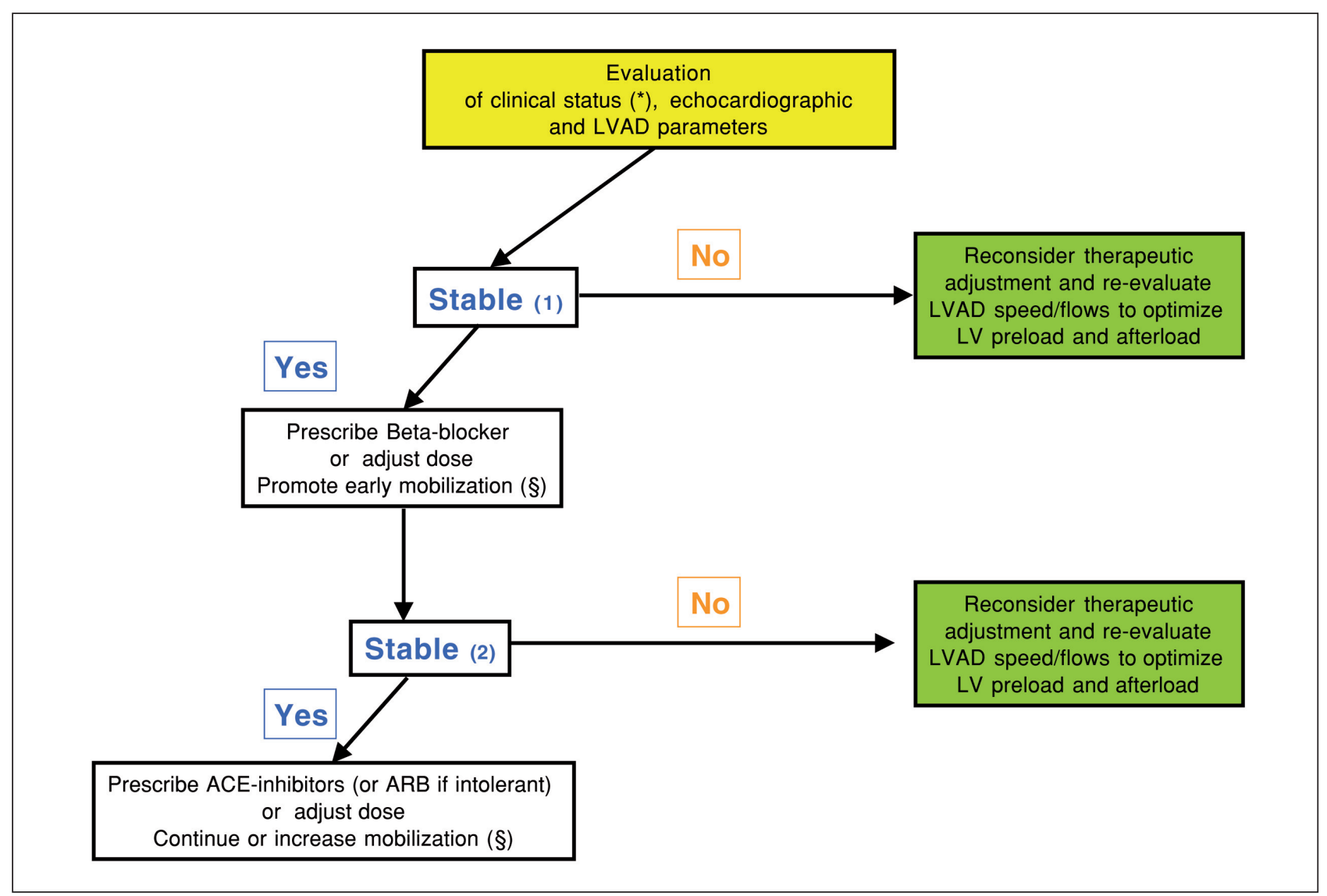

Figure 1. - Proposed in-patient cardiac rehabilitation "step up" pharmacological and non-pharmacological program, developed in our Institution.

(*) Clinical status is defined according physical examination and vital signs, especially mean blood pressure and heart rate values, weights changes, daily diuresis and body temperature.

(1) Stability is when a euvolemic clinical status is achieved with the lower diuretic daily dose.

(2) Clinical stability is defined if no new symptoms and/or changes in vital signs and/or in weight measurements have been observed over one week time.

(§) the early mobilization 10 steps program is reported in detail in Figure 2. 
the mean BP. There appears to be a predisposition for patients to develop hypertension post-VAD implant and management of this is crucial. We strive to maintain mean BP between 70 and $90 \mathrm{mmHg}$ to prevent end-organ damage and to lower possible risks of cerebral bleeds. In the case of hypotension, we recommend to adjust vasoactive medications or consider volume expansion; in the presence of hypertension, a decrease of afterload with medications and volume overload with diuretics are recommended.

\section{Management of volume status and hydration}

It is common for VAD patients to become dehydrated as they continue to limit their oral intake and maintain a salt-restricted diet. To help monitor volume status after discharge, patient self-care routines should include daily charting of weight with attention to symptoms such as orthostatic hypotension. Patients should be prompted to call if their weight fluctuates more than $1.5 \mathrm{~kg}$ during a 24-hour period. An echocardiogram study is recommended to verify that the pump is set at the proper speed [8].

\section{Management of arrhythmias}

Patients in atrial flutter or fibrillation may receive mechanical support, but maintenance of a sinus mechanism is preferred [8]. Ventricular arrhythmias are not uncommon in VAD patients, especially those with an underlying ischemic cardiomyopathy [9]. Often, ventricular tachycardia (VT) or fibrillation may cause change in VAD flow, with relative preservation of cardiac output and consciousness [10]. These arrhythmias, however, are associated with a more malignant course and increased mortality risk and the use of implated cardioveter defibrillators (ICD) is recommended [11]. With axial flow pumps, excessive ventricular unloading leading to suction of the left ventricular wall or septum into the draining cannula can induce VT. This is the most common cause of VT in axial pumps and ceases after clearance of suction [12]. If VT is suspected, an echocardiogram should be performed.

If repeated or sustained ventricular arrhythmias occur, the following steps should be taken: 1) decrease pump speed if arrhythmias occur with suction events, 2) evaluate pump speed under echocardiography for excessive unloading or for contact between the inflow cannula and LV wall (if there is evidence of a decrease in flow, or changes in power, or pulse index), 3) assess carefully the right ventricular (RV) function, 4) consider anti-arrhythmic medications, 5) consider cardioversion.

Cardioversion or defibrillation is possible with all technologies, as is intubation. When external defibrillation is required, the VAD system controller should be disconnected before delivering the shock to avoid electronic disruption. Cardiac arrhythmia should not be treated in an asymptomatic individual if the device is functioning properly. Chest compression may lead to dislodging of the tubing or damage to the device itself. Most importantly, if it is possible, emergency personnel, the patient and caregivers should be educated on the therapies that can or cannot be delivered [13].

\section{Ventricular function and VAD support}

\subsection{Left ventricular function}

A discussion of device-specific speed/flow management for all VAD models and manufacturers is beyond the scope of this paper. In general, care strategies aim to set device speeds/flows to optimize left ventricular preload and afterload while simultaneously avoiding perturbation of RV wall stress. Continuous flow devices (in particular centrifugal pumps) are afterload sensitive and a tight blood pressure control is needed to facilitate optimal device flows and reduce device power consumption. Echocardiography is fundamental to guide left VAD speed/flow optimization. Device settings should allow for decompression of the left ventricle (LV), leading to a reduction in left ventricular volumes and/or dimensions from pre-implant measures. With appropriate left VAD flows/speeds, left ventricular afterload is reduced and, consequently, mitral regurgitation should be insignificant. The apical four chamber view is useful for visualization of the interventricular septum and the left ventricular inflow cannula. Leftward displacement of the septum induced by high left VAD inflows should be avoided due to its impact on RV wall stress and risk of device-induced suction events. Doppler interrogation of the inflow cannula should be without turbulence. Elevated inflow velocities could indicate device thrombosis. A sign of poor LV unloading in some patients is the degree of mitral regurgitation (MR): if the patient has severe MR, consider increasing the pump speed and evaluating inflow and outflow position [8].

Finally, there is growing evidence that aortic insufficiency tends to progress with duration of left VAD support, possibly due to left VAD-induced shear-stress damage to the aortic root and the root side of the aortic valve [14-15]. Although the clinical impact of aortic insufficiency on left VAD outcomes is not yet known, it could lead to ineffective left VAD output through blood recirculation. Thus, aortic insufficiency should be monitored in patients on left VAD support and device speeds may need to be adjusted accordingly if clinical HF is noted with aortic insufficiency progression. There are data to suggest that a fully opening aortic valve may have less propensity for developing regurgitation [15]. Up to now, it is unclear whether optimizing device speeds to ensure regular valve opening can prevent the development of aortic insufficiency. A regularly opening valve will reduce the likelihood of leaflet fusion, and maintenance of normal aortic valve operation is likely important for those in whom myocardial recovery is anticipated. The risk of development of aortic root thrombosis is also lessened in the setting of intermittent aortic valve opening.

\subsection{Right ventricular function}

General considerations for evaluating and managing RV function and setting the pump speed in the post-operative setting include to avoid setting the pump speed so high that it causes a significant leftward septal shift and abnormal RV geometry, which 
can adversely affect RV function. High pump speeds also can collapse the LV and obstruct flow through the LVAD inlet cannula draining the LV. If a patient is clinically decompensating, with signs of poor forward flow or right heart failure, a repeated echocardiogram is recommended to reevaluate pump speed and exclude possible tamponade. When the pump speed is set, the RV should be assessed to see if it becomes dilated and hypocontractile at high or low speeds. Increasing pump speed is crucial, because once the RV begins to show signs of failure it can be very difficult to reverse.

\subsection{Device management}

Pump operating parameters are not surrogates for monitoring the patient's clinical status. Changes in all the parameters must be taken into account when assessing a situation. Owing to variability between patients, trends observed in the system parameters are usually of more value than the absolute values presented. Abrupt changes in the parameters not associated with normal physiologic changes (i.e. exercise) can be used to identify conditions that warrant further evaluation. The use of VAD parameters in troubleshooting selected complications is presented in dedicated reviews $[8,13]$.

\section{Infection}

The Achilles' heel of VAD therapy is infection. Until a fully implantable technology is available, infection will remain the largest obstacle to the success of truly long-term VAD support. Infections occur in $18-59 \%$ [8, 16-18] of VAD patients. HF severity and duration, advanced age, comorbid disease, immunosuppression, suboptimal nutrition, presence of indwelling catheters, and prolonged hospital stay are factors that could contribute to an overall increased risk of infection in VAD patients [20]. Left VAD-related infection can involve every aspect of the device: the surgical site, the driveline, the device pocket, or the pump itself. More than half of all VAD-related infections include multiple sites [20]. In general, driveline infection is the most common, and most VAD-related infections occur between 2 weeks and 2 months after implantation: in most cases, the risk of driveline infection peaks earlier than pump pocket infection, and the cumulative risk of infection increases with duration of VAD use [20]. Antimicrobial prophylaxis administered in the perioperative period is targeted at culprit Gram-positive organisms (Staphylococcus species) as well as certain Gram-negative (Pseudomonas, Serratia) and fungal (Candida) pathogens. The duration of postoperative antimicrobial therapy and the specific regimens administered differ across VAD institutions. Most will continue oral therapy until full driveline incorporation, which occurs between 3 and 6 months postoperatively.

The driveline exit site requires daily disinfection and dressing changes with aseptic technique. If necessary, dressing changes should be done more frequently to keep the wound dry and clean. Some sources recommend silver-impregnated dressings [18]. Stabilization of the driveline is also important.
Abdominal binders are often used [21] while additional gauze, tape, sutures, or stoma-adhesive devices are also recommended [22]. Education of the patient to avoid driveline trauma and to alert the care team if the driveline becomes unstable may also be helpful.

Although infection remains a common complication of VAD placement, there is evidence that the infection rate is declining. A study comparing data from the REMATCH trial to the outcome of patients on destination therapy following market approval of the HeartMate left ventricular assist device demonstrated that the rate of sepsis had decreased from 0.60 to 0.46 events per patient-year and the rate of driveline/pocket/device infection had dropped from 0.54 to 0.38 events per person-year since 2001 [23]. These decreases may be due to improved patient selection and management, including better antimicrobial prophylaxis and improved devices (e.g. smaller size, more durability). In addition, improved outcomes after the REMATCH trial may reflect physician experience, as evidenced by a lower incidence of sepsis at specific investigative centers during the trial [23]. Despite these improvements, the rate of device-related infection still remains unacceptably high and limits the outcome of long-term therapy in the treatment of end-stage HF.

Tables 1 and 2 report the management protocol for driveline infection prevention and care in in-patient cardiac rehabilitation [24-25], based on the experience of our Institute.

\subsection{Ventricular assist device-related endocarditis}

When the inner components of the ventricular assist device are infected, a VAD-related "endocarditis" is established. VAD patients frequently have bacteraemias and fungaemias, as a result of coincidental nosocomial infections such as catheter-related infection, pneumonia and urinary tract infection. Infections arising from other parts of the device, such as the pump pocket and driveline exit site, may also lead to bacteraemia or fungaemia [20]. The probability of an organism in the bloodstream adhering to the ventricular assist device and causing VAD-related endocarditis depends on the characteristics of the device surface, the amount of turbulent blood flow in the device, and the adherence properties of the organism [26]. Device design may help reduce susceptibility to VAD-related endocarditis e.g. rotary (axial) pumps with continuous rather than pulsatile flow may have lower infection rates [27].

\section{Bleeding risk versus thrombosis and thrombotic complications}

Anticoagulation and antiplatelet therapy are required for most VADs due to the potential for in-situ device thrombus formation and cardio-embolic complications. INR therapeutic range is recommended by the VAD manufacture: if the VAD patient is exposed to the risk for ischemic event, the upper limit of INR range should be pursued while for those at risk for hemorrhagic episodes, the lower limit should be the reference.

For the HeartMate II device, rates of device thrombosis in the major trials were very low $(0.02-$ 
Table 1. - Prevention of infection during in-patient cardiac rehabilitation: monitoring and management

1. A photograph of the driveline exit site is taken at the first check-up and at each successive check-up if the following aspects are present:

a. signs of infection

b. tissue in-growth

c. drainage

d. changes in surrounding tissue

2. Flush with saline water and wash with chloroxidine

3. Dressing over driveline exit site must be kept clean and dry

4. Sterile dressing to driveline exit site is changed at least daily using a strict aseptic technique, with the collaboration of two nurses:

a. dressing is changed several times a day if drainage is observed

b. if dressing is clean and dry without local signs of infection, covering replacement is postponed, with a maximum of one week between dressing changes

5. Driveline fixation: proper positioning and sizing of the abdominal binder to prevent driveline lesion or disconnection

Table 2 - Recommended management plan for driveline infection during in-patient cardiac rehabilitation

\begin{tabular}{|c|c|c|c|c|c|c|}
\hline & $\begin{array}{l}\text { Local signs } \\
\text { of infection }\end{array}$ & Cultures & Drainage & $\begin{array}{c}\text { Fever and/or } \\
\text { positive blood tests }\end{array}$ & $\begin{array}{c}\text { Blood } \\
\text { cultures }(\&)\end{array}$ & Treatment \\
\hline Grade 0 & No & No & No & No & No & Saline water, chloroxidine \\
\hline Grade 1 & Yes & No & No & No & No & Silver impregnated dressing \\
\hline Grade 2 & Yes & Yes & Yes & No & No & $\begin{array}{c}\text { Oral }(*) \text { or intravenous antibiotics }(\S) \\
\text { Expose device to allow for constant drainage } \\
\text { Pain relief if required }\end{array}$ \\
\hline Grade 3 & Yes & Yes & Yes & Yes & Yes/No $(\dagger)$ & $\begin{array}{c}\text { Intravenous antibiotics } \\
\text { Surgical implantation of antibiotic beds } \\
\text { Expose device to allow for constant drainage } \\
\text { Pain relief if required }\end{array}$ \\
\hline \multicolumn{7}{|c|}{$\begin{array}{l}\text { Local signs of infections at the driveline exit site: redness/erythema, warmth, tenderness/induration, tissue in-growth, and pain } \\
(\&) \text { : preferably two or more. }(*) \text { Wide spectrum antibiotics or related to the results of exit site cultures. ( } \$ \text { ) Daptomicin if } \\
\text { Methicillin-Resitant Staphylococcus Aureus infection [24-25]. ( } \dagger \text { ) if blood cultures are positive, duration of antibiotics treatment, } \\
\text { screening evaluation (i.e. transesofageum echocardiogram, abdomen TC and so on) and clinical surveillance should be adapted to } \\
\text { clinical situation. }\end{array}$} \\
\hline
\end{tabular}

0.03 events/patient-year), with rate of ischemic stroke ranging from 0.06 to 0.13 events/patient-year $[2,4]$. No device thrombosis occurred in the XVE arm of the HeartMate II Destination Therapy trial, and ischemic stroke rates were 0.10 events/patientyear [2]. In the outpatient setting, close monitoring of INRs, serum lactic acid dehydrogenase, serum free hemoglobin, bilirubin, and hematocrit levels is important.

Acquired von Willebrand syndrome with a reduction in von Willebrand factor (vWF) high molecular weight multimers has been well characterized and tends to have an early onset (as soon as $24 \mathrm{~h}$ ) after VAD support, resolving upon device explant [2831]. Other VAD-induced changes in the coagulation system include a reduction in factors XI and XII and an increase in markers of fibrinolysis [30]. In the major trials, bleeding requiring blood product transfusion occurred in $42-81 \%$ of patients and bleeding requiring surgery occurred in $15-30 \%$ of VAD patients $[1-2,4]$. Hemorrhagic stroke rates range from 0.05 to 0.11 events/patientyear $[1-2,4]$.
Of growing concern are complications from gastrointestinal bleeding. Similarly to Heyde's syndrome in aortic stenosis, patients on VAD support can develop gastrointestinal arteriovenous malformations with high propensity for bleeding due to acquired vWF deficiency. Cohort studies show a cumulative incidence of gastrointestinal bleeding ranging from $32 \%$ to $40 \%$ with a mean time to first bleed of 112 and 87 days, respectively [28, 31-32]. Angiodysplastic bleeds can occur anywhere in the gastrointestinal tract, tend to be recurrent, and carry associated burdens of blood transfusion and allosensitization risk. Compared with pulsatile flow devices, the risk for angiodysplasia development appears to be higher in patients supported with continuous flow VADs. Unfortunately, there is no known intervention to prevent or reduce gastrointestinal bleeding risk.

\section{Discharge plan; patient and family education}

Successful discharge planning begins preoperatively, with assessment of the patient's cognitive 
abilities, support system, home environment, and financial considerations $[8,13]$. Device education and self-care management should be completed by the VAD team. In addition to the extensive in-patient education provided after VAD implant, re-education in the outpatient setting is also crucial.

Patients and caregivers should receive frequent reviews on

1) device alarms; patients should be educated to record VAD information every day on a chart, that assists them in knowing what their "normal" VAD parameters are;

2) proper battery management: changing batteries, carrying back up batteries, purchasing an electric generator for emergency use;

3) aseptic driveline care and occlusive bandag^ing;

4) driveline fixation: proper positioning and sizing of the abdominal binder, proper fixation of leads to avoid fracture, high-risk activities that may increase driveline infection risk;

5) controller fixation;

6) signs and symptoms of gastrointestinal bleeding. In practice, the discharge of a VAD patient is a complex and time consuming procedure: discharge criteria from the cardiac rehabilitation program are listed in table 3; in addition, several care organization items need to be tackled before the patient leaves the hospital (table 4).

Although the VAD coordinator (see below) is usually the person charged with training patients and their family members/caregivers, and assuring follow-up throughout VAD support, all HF team members have valuable contributions to make when preparing the patient for discharge. When appropriate, the patient's primary care physician should be involved, and communication among all team members should be coordinated $[8,13]$.

Table 3. - Discharge criteria from the in-patient cardiac rehabilitation

1. Recovery from the surgical procedure

2. Healing of surgical wounds

3. Stabilized fluid balance

4. Optimization of cardiovascular pharmacological therapy

5. Stabilized INR and anti-coagulant dose

6. Regular functioning of VAD: see "optimized" range of VAD parameters

7. No visible and clinical signs of infection at the driveline.

8. Stable clinical and functional status

9. Physical and respiratory therapy rehabilitation completed

10. Stable psychological status

11. Proficiency in the management of the equipment

12. Patient physically able to manage own care (or most of it)

13. Readiness of the patient's home

\section{Psychosocial issues and end-of-life concerns}

Before VAD implantation, patients must be screened for emotional and psychological readiness, family and social support, and home safety [33-34]. Family members or friends should be aware that the demands on them will be great. VAD patients face a unique set of challenges including the loss of independence, concern with burdening caregivers, fear of the complexity of managing the device or related equipment, change in family dynamics, strain on finances, and fear of dying. Poor adherence to medicine intake and follow-up, for instance, could be an expression of anxiety or of anger, irritability, agitation, frustration, intolerance, sleep disturbances or nightmares. If a VAD patient manifests such behaviors, it might be advisable to try to get them to talk about their traumatic experiences and possibly to consult a psychologist [35].

Finally, perhaps more so than for any other form of therapy, with VAD implantation there must be a clear understanding among the patient, family, and healthcare providers of end-of-life issues. VAD technology has the ability to maintain stable hemodynamics despite difficult and unanticipated complications that the patient, family, and healthcare providers do not wish to suffer. To avoid confusion and in addition to a conventional will, the patient's and family's end-of-life desires should be documented prospectively. Such documentation should include the circumstances under which the VAD will be turned off, which would result in almost certain death.

\section{Living with a VAD}

Although left VAD support is not without complications, patients spend most of their time outside the hospital setting. As outpatients, they may resume many of their previous normal activities, but some restrictions are necessary to ensure patients' wellbeing and optimal device function. Due to the sensitive nature of these machines, patients should avoid extremes of temperature for prolonged periods of time [13]. Because VAD patients remain permanently susceptible hosts to infection, they should be cautious in surroundings that can place them at a greater risk (e.g. day care facilities, contact with sick individuals, crowded living conditions, poor hygiene). It is recommended that individuals avoid power stations and power lines for possible electrical interference $[8,13]$.

\section{A multidisciplinary approach: role of cardiovascular prevention and rehabilitation programs}

Adequate cardiac rehabilitation (CR) including physical, occupational, and nutritional therapy is a central part of the patient's recovery from VAD implantation [13]. Although most CR facilities are unwilling to take VAD patients due to a lack of training or knowledge about the technology, this can be overcome with a strong collaborative relationship [36]. Since adequate exercise performance for daily life activities is a mandatory pre-requisite for per- 
Table 4. - Discharge plan: check list (Check every item before discharge)

- Review VAD functioning and alarms

- Inspect surgical site and driveline for infection: take a photograph of the driveline exit

- Review VAD functioning and alarms with the patient and caregivers

- Review VAD management and troubleshooting with the patient and caregivers: provide normal range of VAD parameters and a sheet for recording daily VAD parameters

- Provide the therapeutic INR range and an INR summary sheet with values and discharge planning

- Provide information about the management of sub-optimal INR

- Explain the medical regimen to patient and caregivers

- Alert the local electric utility company of the presence and necessities of a VAD patient

- Identify harmful environments at home and outside

- Provide advice for safe living with VAD

- Provide emergency contact mobile telephones numbers and explain how to get in touch with the VAD team

- Contact the VAD coordinator of the department of cardiovascular surgery, responsible for the patient's VAD implantation: provide him/her an updated description of patient's clinical and functional status

- Notify the primary care physician about the patient's clinical and VAD management details

- Notify the local first responders and emergency department personnel about clinical and VAD management details

- Notify the cardiology department of the reference hospital about clinical and VAD management details

manent VAD support, an intensive multidisciplinary cardiac rehabilitation (CR) program has the potential to both increase exercise performance and improve quality of life. After VAD implantation, recipients should initiate an exercise program as soon as possible, based on a series of sequential exercise steps [34]. Criteria for beginning the exercise program or interrupting the exercise session, which should be standardized, are reported elsewhere [37].

We have developed an individualized functional evaluation scheme, including echocardiogram, 6minute walking test and cardiopulmonary exercise test, according to an early mobilization "10 step up" exercise program (figure 2): the initial step, the modality and intensity of each exercise session as well as the progression of the overall program is generally evaluated by the VAD coordinator in conjunction with the physical therapist. Clinical status and functional abilities screening are fundamental prerequisite for exercise progression in the individual LVAD patient.

A multidisciplinary $\mathrm{CR}$ and preventive approach to VAD patient care includes cardiac surgeons, HF specialists, infectious disease consultants, physiotherapists, exercise physiologists, psychologists, dietitians, and social workers [8, 13, 36, 38]. In the team, if possible, a specialized cardiologist should be the VAD coordinator [38], and should be knowledgeable about VAD-related cardiac physiology at rest and during exercise, including the interpretation of hemodynamic data and responses to pharmacological manipulation. In addition, the coordinator should be trained in the following: 1) mechanical problems related to the VAD, valve conduit, right ventricular dysfunction or ischemia, tricuspid regurgitation, aortic regurgitation with shunting of VAD output, pulmonary vascular resistance, systemic vascular resistance, and patent foramen ovale with hypoxemia, 2) interpretation of LVAD-related hemodynamics based on noninvasive, invasive, and console data, 3) supraventricular and ventricular arrhythmias pertinent to the device, such as suction events with axial flow devices, 4) hematological issues, including bleeding and thrombosis associated with antiplatelet and anticoagulant therapy, and device-related hemolysis, 5) infectious complications, 6) common symptoms and signs-dyspnea, fatigue, fever, anemia, 7) device alarms, 8) physiological and device-related factors affecting longevity, 9) nutritional deficiency, 10) psychosocial status and quality of life, 11) right-heart catheterization data, 12) the role of exercise and rehabilitation therapy.

The health care professional figures, working in a team tactic, in our in-patient $\mathrm{CR}$ and prevention program are reported in figure 3: while many tasks can be shared by more than one team member, some tasks require specific skills and expertise and should be performed by the appropriate, designated health professional. Team members may have different backgrounds and training and therefore different areas of expertise. To avoid overlapping of roles, we have determined in advance those tasks which should be undertaken by a designated team member and those which may be shared by several team 


\begin{tabular}{|l|c|}
\hline $\begin{array}{l}\text { Early mobilization; } \\
\text { the } \mathbf{1 0} \text { steps protocol }\end{array}$ & $\begin{array}{c}\text { Timing of echocardiographic and } \\
\text { functional evaluations }\end{array}$ \\
\hline Positioning & -- \\
\hline Bed mobility activities & -- \\
\hline Transfers from bed/in room, sitting exercise & -- \\
\hline Cycling without load in sitting position & -- \\
\hline $\begin{array}{l}\text { Gait on a surface (with or without rolling } \\
\text { walker) }\end{array}$ & 6-Minute WALKING test \\
\hline Begin stair climbing & \begin{tabular}{c} 
Echocardiogramm \\
\hline Unloading cycling on stationary bicycle
\end{tabular} \\
\hline Arm ergometry & -- \\
\hline Treadmill & -- \\
\hline Loading cycling & $\begin{array}{c}\text { Pre-discharge functional evaluations } \\
\text { 6-Minute WALKING test } \\
\text { or/and }\end{array}$ \\
\hline & Cardiopulmonary exercise testing \\
\hline
\end{tabular}

Figure 2. - Timing of echocardiographic and functional evaluations according to the progression of the 10 step mobilization program. See text for explanation.

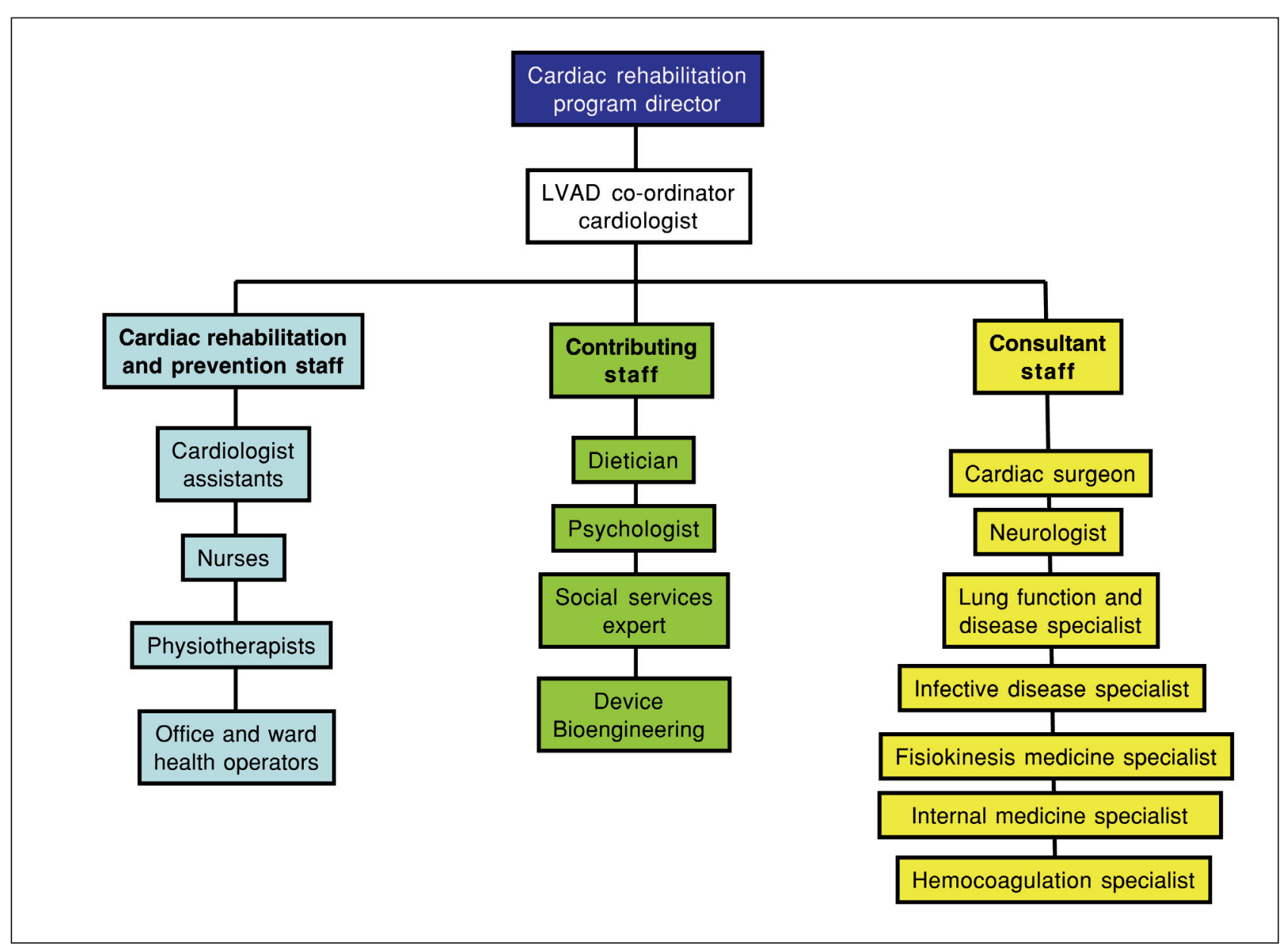

Figure 3. - An examples of health care personnel organizational chart involved in an in-patient cardiac rehabilitation setting, derived from the experience developed in our Institution. 
members. Finally, team meetings to facilitate communication between team members and to provide regular opportunities to discuss patients who have been recently enrolled or those who are experiencing problems are programmed on a regular basis.

The program director should be a cardiologist with good organizational, management and interpersonal skills, while the LVAD coordinator is a cardiologist that is familiar with both VADs and surgical interventions and must have experience regarding pulsatile versus axial/continuous flow devices, intracorporeal versus extracorporeal support, left ventricular versus biventricular or total artificial heart support, and with the mechanical problems related to the VAD as well as alarms interpretation. A good interrelationship between these specialists is essential to ensure efficient running of the rehabilitation program.

A proactive approach to outpatient VAD care can help identify problems earlier with more effective treatment $[8,13]$. The following aspects of long-term management warrant consideration: 1) the frequency of follow-up clinic visits varies among patients, depending on medical issues, concerns for selfcare; 2) patients should return to the clinic weekly until the VAD team determines that less frequent visits are prudent; 3 ) follow-up by telephone calls may be useful in maintaining a constant communication with VAD patients, especially those who do not live near the implant or rehabilitation center; 4) anti-coagulation therapy must be carefully monitored; 5) outpatients should be assessed whenever significant changes in pump function are observed and after any alarm condition: 6) changes in exercise tolerance, weight, appearance of the percutaneous lead exit site, or other symptoms should be considered as potentially serious adverse events, and a thorough evaluation should be promptly performed; 7) outpatients must have access to the VAD team at all times and have the appropriate contact information in case of emergency or for questions and technical support; 8) serial echocardiograms with a ramped speed study during routine clinic visits may be useful to reassess the pump speed setting and to evaluate the potential of myocardial recovery [8].

\section{Conclusion}

VAD therapy offers patients with advanced HF improved survival and quality of life. It is developing into a reliable therapeutic option and, for some patients, the VAD will in all likelihood enable them to delete the first two letters of the word "incurable". The current task is to promote awareness of the benefits of this technology in the greater cardiology community. We believe that the most powerful tool might be the patients themselves since, as more patients receive VADs and return to their communities with improved quality of life, they will serve as ambassadors. The development of dedicated cardiovascular prevention and rehabilitation programs might further help to raise awareness about the benefits of VAD therapy.

Acknowledgments: The authors are grateful to Rosemary Allpress for her careful revision of the English manuscript.
We are in special debt to the late Dr Enzo Bosimini who, as the first of our team in charge of managing patients with mechanical circulatory support, passed on to us his knowledge and expertise, stimulated our research curiosity, and created the existing platform for us to work together as a team.

In addition, we would like to thank Prof. Giuseppe Faggian and Dr Alberto Forni of Cardiochirurgia Universitaria, Ospedale Civile Maggiore di Verona Borgo Trento, Italy; Prof. Mauro Rinaldi and Dr. Paolo Centofanti of Cardiochirurgia Universitaria, Ospedale S. Giovanni Battista di Torino, Italy; Dr. Federico Pappalardo of the Rianimazione Cardiochirurgica, and Dr. Michele De Bonis of the Cardiochirurgia, IRCCS San Raffaele Milano, for their effective and most valuable collaboration.

Finally, we are obliged to Ing Silvia Scuri, Dr. Emilio Contini (Artech s.r.l., "Jarvik Italia"), Dott. Annalisa Rastelli, Dott. Mauro Cotza ("THORATEC Hear Mate Italia"), Ing. Antonella Marino, and Claudio Gibelli (Vega s.r.l., "Berlin Hear Italia") for their passionate technical support.

\section{References}

1. Rose EA, Gelijns AC, Moskowitz AJ, et al. Long-term mechanical left ventricular assistance for end-stage heart failure. N Engl J Med 2001; 345: 1435-43.

2. Slaughter MS, Rogers JG, Milano CA, et al. Advanced heart failure treated with continuous-flow left ventricular assist device. N Engl J Med 2009; 361: 2241-2251.

3. Pagani FD, Miller LW, Russell SD, et al. Extended mechanical circulatory support with a continuous-flow rotary left ventricular assist device. J Am Coll Cardiol 2009; 54: 312-21.

4. Miller LW, Pagani FD, Russell SD, et al. Use of a continuous-flow device in patients awaiting heart transplantation. N Engl J Med 2007; 357: 885-96.

5. Lund LH, Matthews J, Aaronson K. Patient selection for left ventricular assist devices. Eur J Heart Fail 2010; 12: 434-443.

6. Miller LW, Lietz K. Candidate selection for long-term left ventricular assist device therapy for refractory heart failure. J Heart Lung Transplant 2006; 25: 756-764.

7. Jessup M, Abraham WT, Casey DE, et al. 2009 focused update: ACCF/AHA Guidelines for the Diagnosis and Management of Heart Failure in Adults: a report of the American College of Cardiology Foundation/American Heart Association Task Force on Practice Guidelines: developed in collaboration with the International Society for Heart and Lung Transplantation. Circulation 2009; 119: 1977-2016.

8. Slaughter MS, Pagani FD, Rogers JG, et al for the HeartMate II Clinical Investigators. Clinical management of continuous-flow left ventricular assist devices in advanced heart failure. J Heart Lung Transplant 2010; 29: S1-S39.

9. Bigger JT Jr., Fleiss JL, Kleiger R, Miller JP, Rolnitzky LM. The relationships among ventricular arrhythmias, left ventricular dysfunction, and mortality in the 2 years after myocardial infarction. Circulation 1984; 69: 250-8.

10. Oz MC, Rose EA, Slater J, Kuiper JJ, Catanese KA, Levin HR. Malignant ventricular arrhythmias are well tolerated in patients receiving long-term left ventricular assist devices. J Am Coll Cardiol 1994; 24: 1688 -91.

11. Bedi M, Kormos R, Winowich S, McNamara DM, Mathier MA, Murali S. Ventricular arrhythmias during left ventricular assist device support. Am J Cardiol 2007; 99: 1151-3.

12. Vollkron M, Voitl P, Ta J, Wieselthaler G, Schima H. Suction events during left ventricular support and ventricular arrhythmias. J Heart Lung Transplant 2007; 26: 819-25.

13. Wilson SR, Givertz MM, Stewart GC, Mudge, jr GH. Ventricular Assist Devices. The challenges of outpatient management. J Am Coll Cardiol 2009; 54: 1647-59.

14. Cowger J, Pagani FD, Haft JW, et al. The development of 
aortic insufficiency in LVAD supported patients. Circ Heart Fail 2010; 3: 668-674.

15. Pak SW, Uriel N, Takayama H, et al. Prevalence of de novo aortic insufficiency during long-term support with left ventricular assist devices. J Heart Lung Transplant 2010; 29: 1172-1176.

16. Morgan JA, John R, Rao V, et al. Bridging to transplant with the HeartMate left ventricular assist device: the Columbia Presbyterian 12-year experience. J Thorac Cardiovasc Surg 2004; 127: 1309-16.

17. Robbins RC, Kown MH, Portner PM, Oyer PE. The totally implantable novacor left ventricular assist system. Ann Thorac Surg 2001; 71 (suppl 3): S162-65.

18. Poston RS, Husain S, Sorce D, et al. LVAD bloodstream infections: therapeutic rationale for transplantation after LVAD infection. J Heart Lung Transplant 2003; 22: 914-21.

19. Frazier OH, Rose EA, Oz MC, et al. Multicenter clinical evaluation of the heartmate; vented electric left ventricular assist system in patients awaiting heart transplantation. J Heart Lung Transplant 2001; 20: 201-02.

20. Gordon RJ, Quagliarello B, Lowy FD. Ventricular assist device-related infections. Lancet Infect Dis 2006; 6: 426-37.

21. Holman WL, Park SJ, Long JW, et al. Infection in permanent circulatory support: experience from the REMATCH trial. J Heart Lung Transplant 2004; 23: 1359-65.

22. Holman WL, Pamboukian SV, Blood M, Tallaj JA, McGiffi n DC, Kirklin JK. Managing device infections: are we progressing or is infection an insurmountable obstacle? Asaio J 2005; 51: 452-55.

23. Pasque MK, Hanselman T, Shelton $\mathrm{K}$, et al. Surgical management of Novacor drive-line exit site infections. Ann Thorac Surg 2002; 74: 1267-68.

24. Weis F, Beiras-Fernandez A, Kaczmarek I, et al. Daptomicin for eradication of a systemic infection with methicillin-resistant staphylococcus aureus in biventricular assist device recipient. Ann Thorac Surg 2007; 84: 269-70.

25. Beiras-Fernandez A, Kur F, Kiefer R, et al. Multidrug-resistant gram positive infections in patients with ventricular assist device: the role of daptomycin. Transplantation Proceedings 2009; 41: 2589-91.

26. Baddour LM, Bettmann MA, Bolger AF, et al. Nonvalvular cardiovascular device-related infections. Circulation 2003; 108: 2015-31.

27. Goldstein DJ. Worldwide experience with the MicroMed DeBakey ventricular assist device as a bridge to transplantation. Circulation 2003; 108 (suppl 1): II272-77.
28. Uriel N, Pak SW, Jorde UP, et al. Acquired von Willebrand syndrome after continuous-flow mechanical device support contributes to a high prevalence of bleeding during long-term support and at the time of transplantation. J Am Coll Cardiol 2010; 56: 1207-1213.

29. Heilmann C, Geisen U, Beyersdorf F, et al. Acquired von Willebrand syndrome in patients with ventricular assist device or total artificial heart. Thromb Haemost 2010; 103: 962-967.

30. Geisen U, Heilmann C, Beyersdorf F, et al. Nonsurgical bleeding in patients with ventricular assist devices could be explained by acquired von Willebrand disease. Eur $J$ Cardiothorac Surg 2008; 33: 679-684.

31. Hampton CR, Verrier ED. Systemic consequences of ventricular assist devices: alterations of coagulation, immune function, inflammation, and the neuroendocrine system. Artif Organs 2002; 26: 902-908.

32. Stern DR, Kazam J, Edwards P, et al. Increased incidence of gastrointestinal bleeding following implantation of the HeartMate II LVAD. J Card Surg 2010; 25: 352-356.

33. Wilson SR, Mudge, jr GH, Stewart GC, Givertz MM. Evaluation for a ventricular assist device. Selecting the appropriate candidate. Circulation 2009; 119: 2225-32.

34. Deng MC, Loebe M, El Banayosy A, et al. Mechanical circulatory support for advanced heart failure: effect of patient selection on outcome. Circulation 2001; 103: 231237.

35. Rizzieri AG, Verheijede JL, Rady MY, McGregor JL. Ethical challenges with the left ventricular assist device as destination therapy. Philosophy, Ehitcs and Humanities in Medicine 2008; 3: 1-15.

36. Corrà U, Pistono M, Piepoli M, Giannuzzi P. ventricular assist device patients on the horizon of cardiovascular prevention and rehabilitation. Can we convert difficulties into opportunities? Eur J Cardiovasc Prev Rehabil 2011, in press.

37. Corrà U, Pistono M, Mezzani A, Gnemmi M, Tarrogenta F, Caruso R, Giannuzzi P. Cardiovascular prevention and rehabilitation for patients with ventricular assist device. From exercise therapy to long-term therapy. Part I: exercise therapy. Monaldi Arch Chest Dis 2011, 76: 27-32.

38. ACCF/AHA/ACP/HFSA/ISHLT 2010 Clinical Competence Statement on Management of Patients With Advanced Heart Failure and Cardiac Transplant. J Am Coll Cardiol 2010; 56: 424 -53. 